\title{
Implantable and Semi-Implantable Hearing Aids: A Review of History, Indications, and Surgery
}

\author{
Aline Gomes Bittencourt ${ }^{1}$ Patrick Rademaker Burke ${ }^{1}$ Isabela de Souza Jardim ${ }^{1}$ Rubens de Brito \\ Robinson Koji Tsuji ${ }^{1}$ Anna Carolina de Oliveira Fonseca ${ }^{1}$ Ricardo Ferreira Bento ${ }^{1}$ \\ ${ }^{1}$ Department of Otolaryngology, Universidade de São Paulo, São \\ Paulo, São Paulo, Brazil \\ Int Arch Otorhinolaryngol 2014;18:303-310. \\ Address for correspondence Aline Gomes Bittencourt, MD, \\ Department of Otolaryngology, Universidade de São Paulo, Rua \\ Teodoro Sampaio N 483, Sao Paulo, SP 05405000, Brazil \\ (e-mail: alinegbittencourt@gmail.com; bittencourt@iaorl.org).
}

\begin{abstract}
Keywords

- hearing aid

- hearing loss

- middle ear implant

- fully implantable hearing aid

- Envoy

- Vibrant Soundbridge

Introduction The complaints associated with the use of conventional amplifying hearing aids prompted research at several centers worldwide that ultimately led to the development of implantable devices for aural rehabilitation.

Objectives To review the history, indications, and surgical aspects of the implantable middle ear hearing devices.

Data Synthesis Implantable hearing aids, such as the Vibrant Soundbridge system (Med-El Corporation, Innsbruck, Austria), the Maxum system (Ototronix LLC, Houston, Texas, United States), the fourth-generation of Carina prosthesis (Otologics LLC, Boulder, Colorado, United States), and the Esteem device (Envoy Medical Corporation - Minnesota, United States), have their own peculiarities on candidacy and surgical procedure.

Conclusion Implantable hearing aids, which are currently in the early stages of development, will unquestionably be the major drivers of advancement in otologic practice in the 21 st century, improving the quality of life of an increasingly aged population, which will consequently require increased levels of hearing support.
\end{abstract}

\section{Introduction}

Conventional amplifying hearing aids are associated with several drawbacks, such as occlusion of the external auditory canal, insufficient high-frequency gain for individuals with "ski-slope" hearing loss, feedback, limitations in activities of daily living (inability to participate in water activities and need for constant maintenance, daily cleaning, and battery replacement), and skin conditions. ${ }^{1-5}$ In addition, rejection of conventional hearing aids often involves cosmetic issues and the stigma of inferiority or disability associated with these devices, as their use makes a previously invisible sensory impairment readily apparent. ${ }^{1-5}$

These complaints prompted research at several centers worldwide that ultimately led to the development of implantable devices for aural rehabilitation. These "active" (fully

received

September 3, 2013

accepted

September 17, 2013

published online

April 9, 2014 implantable and semi-implantable) devices bypassed the limitations of sound transmission through the external auditory meatus.

The aim of this article is to review the history, indications, and surgical aspects of the implantable middle ear hearing devices.

\section{Review}

\section{Semi-Implantable Hearing Aids}

\section{Vibrant Soundbridge}

The Vibrant Soundbridge (VSB) system was the first semiimplantable middle ear hearing device to receive U.S. Food and Drug Administration (FDA) approval, namely for use in patients with moderate to severe sensorineural hearing loss. ${ }^{3}$
Copyright $\odot 2014$ by Thieme Publicações License terms Ltda, Rio de Janeiro, Brazil
DOI http://dx.doi.org/ 10.1055/s-0033-1363463. ISSN 1809-9777.

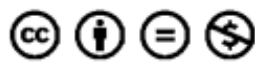


It was initially developed by Symphonix Devices A, Inc. (San Jose, California, United States) and has been manufactured and distributed by Med-El Corporation (Innsbruck, Austria) since 2003. The speech processor has been updated several times, and the device is currently available with the Amadé audio processor.

The VSB comprised two parts: the internal, surgically implanted receiver/demodulator, also known as the "Vibrating Ossicular Prosthesis" (VORP), and the external audio processor, which is placed onto the scalp and remains in place by magnetic attraction between the two parts.

The disk-shaped audio processor has a diameter of $2.5 \mathrm{~cm}$ and is less than $1 \mathrm{~cm}$ thick. It contains two microphones, a microprocessor, and an antenna that transmits signals to the implanted portion of the system. The two microphones pick up sound and convert it to electrical signals, which are then processed and conveyed to the internal portion of the system by magnetic induction. This process consumes power, which is provided by the audio processor battery. The Amade audio processor has wind-noise reduction and sound-smoothing capabilities (reduces loud, unexpected noises) and identifies and reduces background noise without affecting speech signals.

The receiver/demodulator (VORP) comprises an antenna that receives the waveform output of the microprocessor and sends a corresponding electrical current to the floating mass transducer (FMT) through a silicone-coated gold cable. The FMT is a minute $(2 \times 1.8 \mathrm{~mm})$ electromagnetic cylinder placed at the end of the conductor link. Its housing contains a micromagnet that can move or vibrate. The aforementioned gold cable, which connects the VORP to the transducer, is wound around the FMT housing. When the electrical signal of the demodulator is sent through this coil, it generates a magnetic field that moves (vibrates) the FMT micromagnet. The frequency and intensity of this vibration determines the sound generated by the FMT and transmitted to the inner ear.

Since 2008, approved indications for the VSB have expanded to include patients with conductive and mixed hearing loss. ${ }^{4}$ Several trials have demonstrated that placement of the FMT onto vibrating middle ear structures other than the incus can be equally effective. ${ }^{6-9}$

The criteria for VSB implantation are as follows:

- Age over 18 years (in exceptional cases, the system can be implanted in children, and is approved for this purpose in Europe)

- Little or no benefit from conventional hearing aid use or clinical contraindications to conventional hearing aids (e. g., exostoses, recurring otitis externa)

- Malformations of the outer or middle ear

- Bilateral, moderate to severe sensorineural, conductive, or mixed hearing loss that has been stable for at least 2 years

- Sequelae of chronic otitis media, open cavity, absence of the ossicular chain, and otosclerosis with technical contraindications to stapedotomy

Computed tomography (CT) imaging of the temporal bones is mandatory for surgical planning and assessment of the adequacy of the facial recess for device placement (a space of at least $3 \mathrm{~mm}$ is required for passage of the FMT). ${ }^{6,8}$

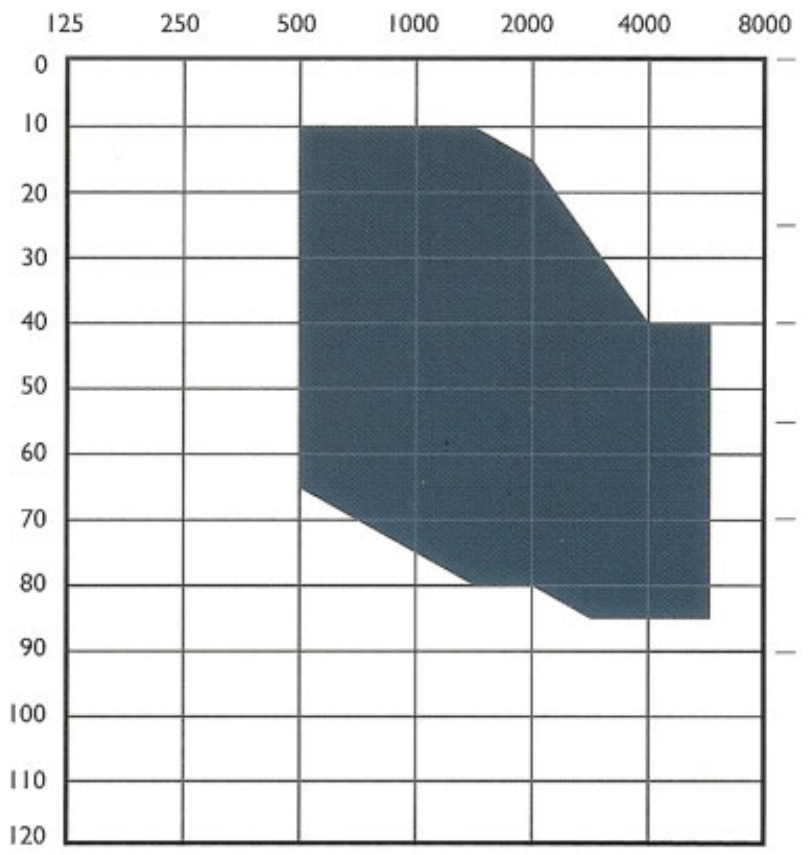

Fig. 1 Bone threshold range for Vibrant candidacy in patients with pure sensorineural hearing loss. Image courtesy of the manufacturer, Med-El Corporation (Innsbruck, Austria).

The FMT may be placed in several different ways, depending primarily on type of hearing loss and anatomical condition of the ear. It may be implanted on the ossicular chain, on the oval window, or on the round window. ${ }^{7,8}$ Patients with sensorineural hearing loss who cannot be satisfactorily fit with a conventional hearing aid or who have recurrent otitis externa are candidates for FMT placement onto the long process of the incus. ${ }^{4}$ - Fig. 1 shows the audiologic indication range for FMT coupling to the incus.

The VSB is particularly helpful when the benefits of conventional amplification are limited for hearing thresholds of 70 to $80 \mathrm{~dB}$; this is the indication for which the VSB was originally developed. ${ }^{3}$ The FMT is attached to the ossicular chain by means of a titanium clip. With this fixation system, the floating-mass magnet remains perpendicular to the long process of the incus. Hence, when the FMT vibrates, this vibration is amplified and relayed to the entire chain, and the stapes in particular transmits this vibration to the perilymph. $^{3}$

Placement is performed under general anesthesia and usually takes 2 hours. Specialist instruments required include a VORP template, forming forceps designed specifically for the FMT clip, and a 7-mm skin flap gauge. The operative technique is as follows:

1. The postauricular area is infiltrated and the incision is marked (Wild's incision).

2. The VORP template is positioned onto the skull at a 45degree angle to the horizontal plane to ensure optimal postauricular placement of the external unit after surgery.

3. The incision is made at least $2 \mathrm{~cm}$ from the edge of the device to reduce the risk of extrusion or infection. 


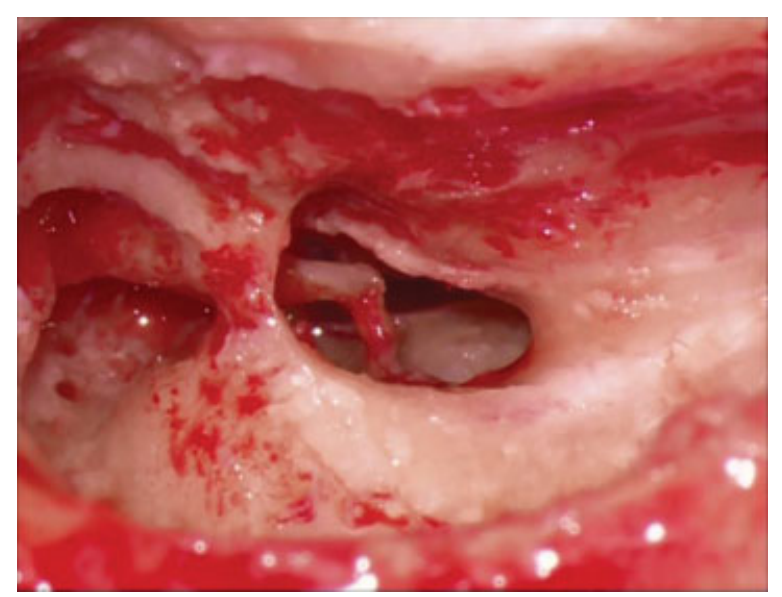

Fig. 2 Posterior tympanotomy for placement of the Vibrant implant (Med-El Corporation, Innsbruck, Austria). The tympanotomy must be large enough to enable passage of the coil, visualization of the long process of the incus, and attachment of the coil clip.

4. An anterior-based periosteal flap is raised with the aid of the 7-mm skin flap gauge, which ensures the flap is thin enough to accommodate the audio processing unit.

5. Once the mastoid is exposed, a simple mastoidectomy and posterior tympanotomy are performed.

6. The posterior tympanotomy must be wide enough to ensure adequate exposure of the middle ear space for FMT placement (-Fig. 2). Visualization of the incudostapedial joint is essential.

7. A seat is fashioned for the VORP device, with the aid of the VORP template placed at a 45-degree angle to the horizontal plane.

8. At this point, the device may or may not be secured with sutures.

9. The FMT clip is opened to an approximate width of $0.5 \mathrm{~mm}$.

10. Once the long process of the incus is exposed, the FMT is carefully placed in contact with the incudostapedial joint, and its attachment clip onto the incus (-Fig. 3). The forming forceps are used to tighten the FMT clip onto

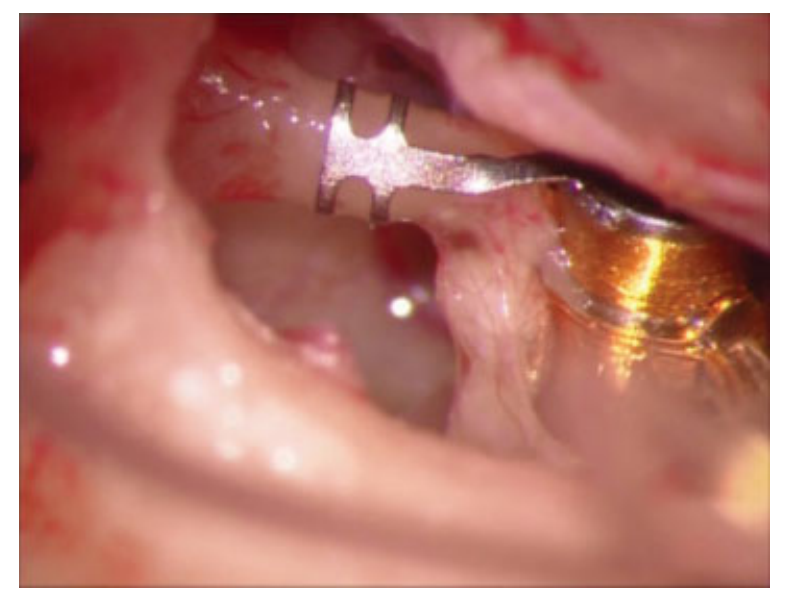

Fig. 3 Site of coil attachment on the long process of the incus. the incus. (Glass ionomer cement may also be used to attach the clip to the long process of the incus.)

11. The FMT is positioned parallel to the axis of the stapes, so that it vibrates directly into the middle ear.

12. Care is taken to prevent the conductor link from contacting the bony surface of the facial recess.

13. Finally, an intraoperative test of the device is performed.

Eight weeks after implantation and once cleared by the surgeon, the patient returns to the audiologist for hearing assessment, and the device is activated.

Advantages of the VSB system include:

- Recovery of auditory function: when indications are precise and surgery is performed properly and successfully, patients benefit from better-than-preoperative inner ear stimulation, which enables patients to better recognize and understand sounds and words. In best-case scenarios, the VSB system enables achievement of hearing thresholds superior to preoperative bone conduction (overclosure of the air-bone gap).

- The external placement of the VSB audio processor ensures that patients can benefit from the most recent technology simply by updating the external portion of the device.

- Open ear canal: there is no occlusion effect. The audio processor is placed onto the scalp and easily concealed by hair.

- No feedback effect: As the VSB does not use a speaker to relay sound, there is no feedback effect, thus enabling better amplification of high-pitched sounds and improving overall hearing quality.

- The system is fully reversible.

- Magnetic resonance imaging (MRI) compatibility: the FMT-coupled ossicular chain remains stable at exposures of up to $1.5 \mathrm{~T}$.

One limitation of the VSB system is its complete inability to improve hearing at the 500-, 250-, and $125-\mathrm{Hz}$ frequencies, regardless of FMT placement on the oval window, round window, or long process of the incus. ${ }^{3,9}$

\section{Maxum}

In 2009, Ototronix LLC (Houston, Texas, United States) introduced the Maxum system, which comprises an external audio processor and an internal implantable device. The external processor may be placed within the canal or behind the ear, as a conventional hearing aid, and consists of a microphone and an amplifier. Electrodes are connected to a custom-made electromagnetic coil, which is placed near the eardrum ( $2 \mathrm{~mm}$ from a magnet implanted at the incudostapedial joint). ${ }^{10}$

The implant itself ( $1.35 \mathrm{~mm}$ in diameter, $2 \mathrm{~mm}$ long) consists of a magnet, its housing, and an attachment collar. A hermetically sealed, laser-crimped titanium canister encases the magnet. A helical collar attached to this canister is used to fixate the implant onto the incudostapedial joint. The collar is placed off-center in relation to the long axis of the canister, thus positioning the implant away from the eardrum and promontory. 
The essential difference between the Maxum system and conventional hearing aids is that the Maxum system employs an electromagnetic processor rather than electroacoustic principles. ${ }^{10}$ Therefore, the tip of the in-ear processor extends deeper into the ear canal than conventional hearing aids and must be placed at a precise, specific angle for optimal results. Hence, there can be no bending or narrowing of the ear canal, and the processor must be made to measure. ${ }^{10}$

The Maxum processor receives sound, amplifies it, processes it according to frequency while maintaining the integrity of the electric signals and relays them directly to an electromagnetic coil in the ear canal. When powered, this coil produces an electromagnetic field that envelops the entire middle ear, inducing the magnet attached to the incudostapedial joint. The magnet then vibrates in perfect synchrony with the original sound waves and causes the stapes to vibrate in harmony with them. Finally, the stapes relays these vibrations directly to the cochlea.

The implantation procedure usually takes 30 to 45 minutes and can be performed under local anesthesia with sedation, both in an outpatient setting and in the operating room.

Briefly, a Rosen incision is made and a tympanomeatal flap is raised to expose the posterior portion of the tympanic cavity. The incudostapedial joint capsule is examined, and the joint is prepared for separation. The incus is slightly elevated to provide gentle traction on the joint capsule, enabling a relatively atraumatic incision and separation of the joint surfaces. A suture thread and blunt-tip needle are used to retract and elevate the incus and to pass the attachment collar around the joint, as follows. ${ }^{11}$

A 4-0 suture with a 1-mm metal sphere on the end is placed at one side of the long process of the incus. The thread is then placed near the oval window niche. A specially designed instrument is used to capture the suture magnetically and pass it to the opposite end of the incus. Gentle traction is placed on the end of the suture, holding the incudostapedial joint taut. The mucosa is then incised and the joint separated posteriorly. At this stage, it is essential that all force used to separate the joint be applied in the anterior direction, in line with the stapedius tendon, so that the latter may stabilize the stapes superstructure. ${ }^{11}$

Once the joint has been opened, the implant is placed onto the end of a nonmagnetic suction tip attached to conventional suction tubing, which is in turn coupled to a Hough-Cadogan pedal to provide precise control of suction pressure. This pedal works by analogy with the gas pedal of an automobile, giving the surgeon full control of the vacuum level during the procedure. Once the implant is in place, it is secured with surgical cement. ${ }^{11}$

The implantation procedure is reversible. ${ }^{11}$ Patients are discharged on the same day with a prescription for an antiinflammatory agent of choice and cleared to return to work on the following day. The implant is activated 4 weeks after surgery to enable complete healing.

The Maxum system is indicated for adult patients (over 18) with moderate to severe sensorineural hearing loss. ${ }^{10}$ It is contraindicated in conductive hearing loss, retrocochlear or central hearing disorders, presence of active middle ear infection, eardrum perforation associated with recurrent otitis media, and incapacitating tinnitus. ${ }^{10}$

\section{Fully Implantable Hearing Aids}

\section{Carina}

The fourth-generation Carina prosthesis is an active, fully implantable middle ear device developed by Otologics LLC of Boulder, Colorado, and is the successor to the first implantable device developed by this company, the semi-implantable middle ear transducer (MET) system. The Carina device is currently approved for use in all European Community countries, and phase II efficacy studies for FDA approval are ongoing.

The Carina system comprises four main components ${ }^{12}$ :

1. The implant proper

2. The implant programming device

3. A remote control

4. A battery charger

The implantable components are:

1. The implant capsule, which contains the implant electronics coupled to a microphone

2. The MET

Two essential components-the microphone and the battery-are housed in the capsule.

Overall, the Carina system works as follows:

1. Sounds are captured by the microphone and relayed to the sound processor within the implant capsule.

2. The sound processor analyses the sound information, amplifies it according to the programmed settings, and converts it into electrical signals that are relayed to the transducer.

3. The transducer then converts these electrical signals into mechanical energy, which causes the target structure (which may be a window or the ossicular chain itself depending on the implantation procedure) to vibrate.

The system contains two microphones housed within a single unit. These microphones are placed in an antagonistic configuration: the external microphone captures environmental sounds, whereas an internal microphone pointed to the midline captures sounds generated by the patient's body (chewing, swallowing).

The remote control, when placed in contact with the coil of the implantable unit, transmits radiofrequency data that enable the user to power the device on and off, change its programming settings, and control the device volume.

The battery-charging system consists of a charging cradle (which is connected to a standard wall outlet), a coil, and a charger, which can be detached from the cradle and coupled to the magnetized implantable unit. Time to charge is 1 hour if the device is charged daily, and each charge lasts 32 hours. According to the manufacturer, the battery lifetime is at least 10 years, after which the entire electronic capsule must be surgically removed for replacement. The MET does not require replacement. 
As other active middle ear devices that contain a magnet, the Carina system is not MRI-compatible, and the device must be surgically removed 2 to 3 weeks before an MRI can be performed.

The Carina system is indicated for patients with sensorineural, conductive, and mixed hearing loss. The criteria for patients with sensorineural hearing loss are as follows. (Criteria for other types of hearing loss have yet to be established clearly.) $)^{12}$

- Minimum age 14 years

- Postlingual hearing loss

- Moderate to severe sensorineural hearing loss, as long as hearing thresholds are within the candidacy range and the three-frequency pure tone average at $500 \mathrm{~Hz}, 1 \mathrm{KHz}$, and 2 $\mathrm{KHz}$ (the mean of the 1-, 2-, 3-, and 4-KHz bands is also acceptable) is in the range of 40 - to $80-\mathrm{dB}$ hearing loss

- An air-bone gap (if one exists) not exceeding $10 \mathrm{~dB}$ in the 500-4 KHz frequencies

- Speech recognition index for monosyllabic words of $40 \%$ or greater at a loudness of $80-\mathrm{dB}$ hearing loss or $40-\mathrm{dB}$ SL in the candidate ear

- Stable hearing thresholds, with no progression or fluctuation or hearing loss on serial audiometric assessment

- Realistic expectations as to the outcome of implant placement

The system is contraindicated in patients with vestibular changes, degenerative bone disorders, middle ear disease, retrocochlear or central nervous system disorders, and prelingual hearing loss. ${ }^{12}$

CT imaging of the temporal bones may be routinely performed in candidates for Carina. The purpose of CT scanning is to assess:

- Integrity of the ossicular chain: presence of an intact ossicular chain enables "textbook" implantation of the system directly onto the body or short process of the incus at the fossa incudis.

- Tegmen mastoideum and tegmen tympani height on coronal slices: these parameters are essential for proper placement of the transducer in the area of the aditus to the antrum, as the MET is $6 \mathrm{~mm}$ high and a space of at least $6 \mathrm{~mm}$ is therefore required.

Briefly, the implantation procedure is performed as follows:

- General anesthesia is induced.

- The postauricular region is infiltrated with lidocaine $2 \%$ with epinephrine $1: 80,000$.

- The implantation site is marked with the aid of a surgical template. The optimal orientation of the electronic capsule is at a 45-degree angle anterior or posterior relative to the vertical axis.

- A 5-cm postauricular incision is made, ensuring adequate exposure for drilling of the mastoid and for safe passage of the entire device (implant/microphone) under zero tension.

- Another incision is made through the muscle and periosteum. This incision is made on a different plane to avoid contact between the first and second incisions at the time of closure.

- A limited mastoidectomy (extending $\sim 2 \mathrm{~cm}$ along the anteroposterior axis from the spine of Henle and 1 to $1.5 \mathrm{~cm}$ superiorly and inferiorly) is performed to allow fastening of the bone bracket, which will support the transducer. A $2.0 \times 1.0-\mathrm{cm}$ template is provided with the surgical kit as a guide.

- Antrostomy and atticotomy are performed to expose the short process of the incus and head of malleus. In the highly pneumatized mastoid, exposure of the malleus is not necessary; the transducer is merely placed at any point along the incus at the fossa incudis.

- Once the target of stimulation along the ossicular chain has been identified, the transducer (MET) bracket is attached. The bracket is secured to the MET provided with the surgical kit, and both are roughly aligned with the site of stimulation and then secured to bone with titanium screws ( 3 or $4 \mathrm{~mm}$ depending on bone thickness). Placement of at least three screws is recommended for proper anchorage. Once the bracket is secured, the mock transducer (template) can be removed.

- A bone bed or recess is drilled out to accommodate the capsule and microphone.

- The electronics capsule is secured to bone with two titanium screws through a loop on each side.

- Two placement options are available for the system microphone: (1) in a bone bed or recess fashioned posteriorly and inferiorly to the bone bracket, as described above. With this placement technique, the microphone is also secured to bone using two titanium screws; or (2) in a subcutaneous pocket at the most superior and posterior point of the sternocleidomastoid muscle, at its attachment at the tip of the mastoid process of the temporal bone. This placement technique is indicated in cases of moderate to severe hearing loss where increased power is required for transduction.

- The transducer is attached definitively to the chosen site of stimulation. Very gently, so as not to damage the MET or injure the ossicular chain, the transducer is positioned onto the secured bracket and fastened so that its tip is no more than $5 \mathrm{~mm}$ from the target site.

- The transducer tip is advanced by micrometric increments until the surgeon visualizes contact and the implantation software detects resistance.

A few intraoperative checks are performed with the aid of a computer software suite (SAFI (Surgical Assistant FIMOS Implant - SAFI, Otologics LLC, Boulder, Colorado, United States)). The results of these checks are filed and later used as a baseline for postoperative verification of implant integrity and function. The manufacturer recommends that the implant be switched on 8 weeks after placement.

\section{Esteem}

In 1984, Yanagihara et $\mathrm{al}^{13}$ attached an implanted piezoelectric device to the head of the stapes to convey signals to the auditory system via induction and began trials of this implant shortly thereafter. ${ }^{14}$ In collaboration with the Rion Company 
Ltda, Japan, the authors conducted clinical trials of a semiimplantable piezoelectric device in over 80 patients with a 10-year follow-up. ${ }^{10}$ After extensive research with semiimplantable devices over the past 15 years, St. Croix Medical, Inc. (now Envoy Medical Corporation - Minnesota, United States) developed the Esteem implant.

The Esteem device is a fully implantable prosthesis now manufactured by the U.S.-based Envoy Medical Corporation. It was granted FDA approval in 2011 and is based on piezoelectric technology. The device is microphone-free; sound is received directly by the eardrum/ossicular chain.

The Esteem piezoelectric system consists of two transducers (sensor and driver) and a sound processor, which contains the device battery. The sensor, which is coupled to the body of the incus with glass ionomer cement, captures mechanical sound waves and transduces them into electrical signals, which are relayed to the sound processor. The driver, which is attached to the head of the stapes, receives this electrical output and converts it back into mechanical energy, which is relayed to an actuator and to the inner ear. The analog sound processor (which also contains the device battery) filters and amplifies the electrical signals received. Overall power consumption is low.

The device is indicated for adult patients with bilateral, moderate to severe sensorineural hearing loss and good speech discrimination (mean aided free-field speech discrimination $>50 \%$ ). The worse ear (that with the worst aided audiologic performance) should be chosen for implantation. ${ }^{15}$

Otoscopic examination must be normal, and CT imaging of the temporal bones must demonstrate sufficient space in the mastoid for transducer placement and a healthy ossicular chain. Temporal bone size plays a critical role in implant placement; poorly pneumatized bones preclude implantation due to the large size of the Esteem device. ${ }^{15}$

Patients with conductive, retrocochlear, or central hearing loss are not candidates for implantation.

Overall, the operative procedure is as follows:

1. A wide mastoidectomy with exposure of the facial recess is performed.

2. The integrity and mobility of the ossicular chain are tested using laser vibrometry (ossicular stiffness makes implantation impossible).

3. The incus and stapes are disarticulated and the long process of the incus is removed.

4. The mucosa overlying the head of the stapes is carefully removed to enable implant attachment.

5. The sensor and driver are secured with glass ionomer cement (-Fig. 4).

6. After implant placement, the entire system is tested and postoperative functional gain is estimated. If gain is deemed inadequate, the implant is repositioned to improve performance.

7. A bone recess is fashioned posterior to the mastoid to house the sound processor.

The device is activated $\sim 45$ days after surgery. The battery has a life span of 7 to 10 years, and can be replaced under local anesthesia through a simple postauricular incision.

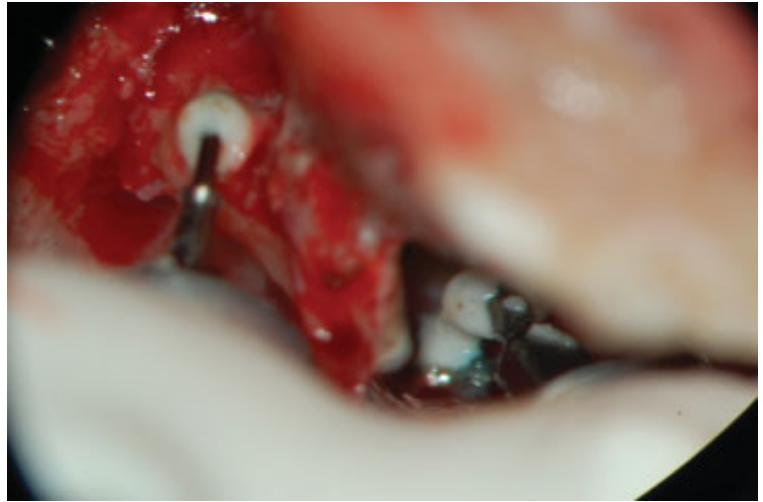

Fig. 4 Esteem system (Envoy Medical Corporation - Minnesota, United States) transducers in place, attached with glass ionomer cement. Intraoperative image.

\section{Discussion}

The first middle ear implants were introduced in 1935, after experiments conducted by Wilska, who placed steel particles onto the tympanic membrane and used an electromagnetic coil placed inside an earphone to generate a magnetic field, which caused synchronous vibration of the steel particles. The eardrum also vibrated, allowing transduction of the resulting sound to the inner ear. In the late 1950s, Rutschmann successfully stimulated the ossicular chain by gluing 10 magnets to the handle of the malleus. This experiment employed an electromagnetic coil. ${ }^{14}$

Proper devices implanted into the middle ear did not appear until the 1970s. ${ }^{14}$ The leading preclinical experiments were those conducted by Fredrickson et al. ${ }^{14}$ Electromagnetic technology was tested in rhesus macaques, and the authors showed that middle ear implants could enable safe and effective transmission of acoustic signals. In their landmark experiment, a magnet was implanted to the head of the stapes and a copper coil placed nearby drove the magnet by electromagnetic induction, creating an auditory signal. ${ }^{14}$

In cooperation with Soundtec Inc. (Oklahoma, United States), Hough et $\mathrm{al}^{11}$ conducted a clinical trial for FDA approval of an electromagnetic device attached to the incudostapedial joint. The processor and electromagnetic coil were embedded in a postauricular or intra-auricular device. After this initial trial, Hough et al $^{10}$ reported increased functional gain, significant improvement in speech recognition in quiet, and comparable discrimination of speech in noise with the SoundTec Direct System. However, although patients preferred the implant to a hearing aid, APHAB (Abbreviated Profile of Hearing Aid Benefit) scores showed a clear drawback of this system: occlusion of the ear canal, which is one of the leading complaints associated with conventional hearing aids. Issues with magnets and unsatisfactory gain eventually led to discontinuation of the SoundTec system and its withdrawal from the market.

After years of trials, two technologies for implantable devices stood out: piezoelectric and electromagnetic. Piezoelectric devices make use of the properties of certain crystals to undergo deformation when exposed to voltage. Electromagnetic devices make use of the movement of metal 
structures attached to the ossicular chain and exposed to a magnetic field.

In 2006, Colletti et $\mathrm{al}^{9}$ described the possibility of removing the FMT clip of the VSB system and placing the FMT directly onto the round window, thus stimulating the cochlear chambers and enabling use of the VSB in patients with conductive and hearing loss. In their study, seven patients with severe mixed hearing loss experienced a postoperative functional gain of 10 to $40 \mathrm{~dB}$, with $100 \%$ speech recognition at $50-\mathrm{dB}$ hearing loss.

Although round-window FMT placement of the VSB system is employed primarily in cases of chronic otitis media and otosclerosis, highly favorable outcomes have been reported in patients with aural atresia. ${ }^{6}$ - Fig. 5 shows the audiologic indication range for FMT placement on the wound window.

In cooperation with Kurz, Med-El introduced a roundwindow coupling system for the VSB system, which consists of a titanium "basket" to which the FMT is coupled, attached to a hemispheric stud. ${ }^{7}$ This hemisphere, which is lodged in the round window seat, is designed to improve signal transmission to the middle ear and can also be used when the surface area available for implantation is reduced or when the FMT cannot be positioned perfectly parallel to the round window membrane. ${ }^{7}$ This reduces the need for drilling of the round window edge, which is advantageous in terms of preservation of the facial nerve and of any residual auditory function. ${ }^{7}$

Oval window placement, which is largely similar to round window placement, is used when surgical access and, particularly, stability of the prosthesis can be ensured by favorable anatomy. In these cases, the FMT can be placed directly onto the stapes footplate or, by means of the attachment clip, attached to the stapes superstructure (if present). ${ }^{6-8}$

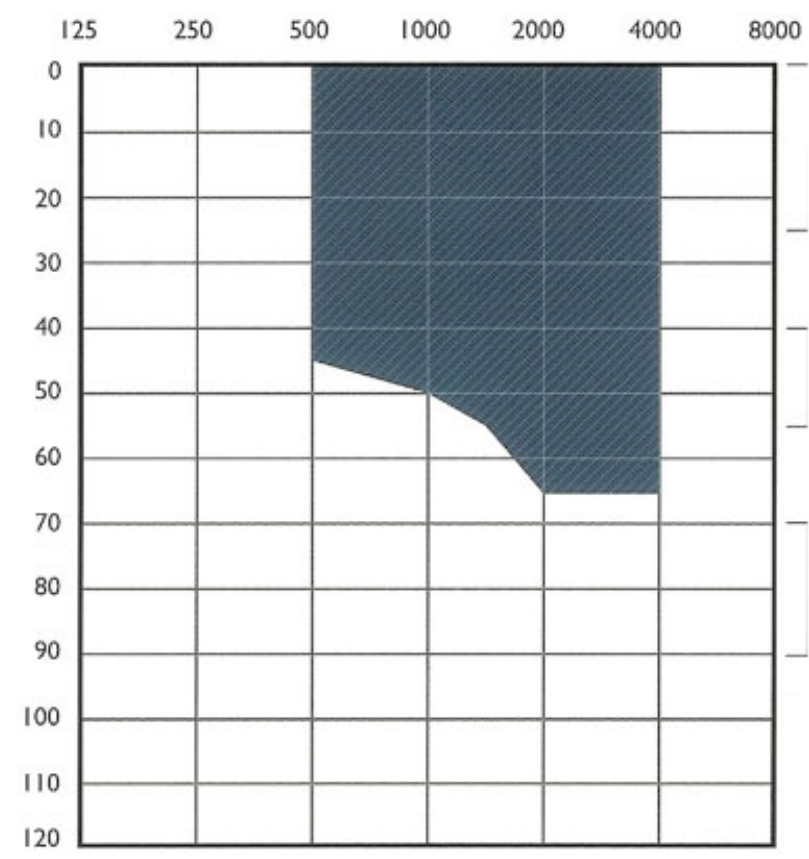

Fig. 5 Bone threshold range for Vibrant candidacy in patients with conductive or mixed hearing loss. Image courtesy of the manufacturer, Med-El Corporation (Innsbruck, Austria).
Other oval window couplers, derived from the partial ossicular replacement prosthesis (PORP) and total ossicular replacement prosthesis (TORP) systems used in passive hearing aids, have also been proposed. ${ }^{7}$ The stability issues encountered with PORP or TORP systems are not a concern in these cases, as the presence of the FMT conductor link makes the FMT far more stable than a PORP or TORP.

Studies of the Maxum device have shown a pure tone gain 7.0- to 7.9-dB superior to that obtained with conventional hearing aids and a mean high-frequency gain of 9.2 to $10.8 \mathrm{~dB}$ as compared with conventional devices. ${ }^{10,11}$ Research has also shown that patients prefer the Maxum system to hearing aids, reporting less intrusive occlusion, improved sound quality, and reduced feedback with the Maxum implant. ${ }^{10,11}$

The Maxum system takes advantage of the amplifying effect of the concha and is the most discrete hearing aid available on the market and the least expensive middle ear implant. It is MRI-compatible up to $0.3 \mathrm{~T}$ and FDA-approved. ${ }^{16}$

Regarding use of the Carina system in patients with conductive and mixed hearing loss, some studies have reported safe and effective outcomes in this setting, with audiologic results superior to those of unaided hearing. ${ }^{2,17}$

It is essential that patients be informed that some noise may be perceived after surgery of the Carina system, particularly when moving the head toward the implant side. According to the manufacturer, the adaptation period is 6 months when the microphone is attached to the skull and 8 to 10 months when it is placed in a soft tissue pocket in the neck region. ${ }^{12}$

The systematic review by Klein et $\mathrm{al}^{2}$ assessed two aspects: safety and effectiveness. Regarding safety, the leading complication was device failure $(n=12,17.6 \%)$. The most common modes of malfunction were charging difficulty and limited battery life ( $n=9$ of 12 ). A single case of device failure was due to disconnection of the transducer from the ossicular chain secondary to head trauma. Partial or complete device extrusion occurred in 4 of 68 patients. Other minor, less frequent complications were functional ossicular chain changes with development of an air-bone gap, otitis media with effusion, vertigo, pain, headache, and aural fullness.

Regarding functional outcomes, the Klein et $\mathrm{al}^{2}$ review found an average improvement in functional gain of $10.4 \mathrm{~dB}$ versus $15.6 \mathrm{~dB}$ with conventional hearing aids (statistically significant). In a phase I trial of 20 patients conducted by Jenkins et $\mathrm{al}^{18}$ to support FDA approval, recognition of monosyllabic words was superior with conventional hearing aids as compared with the MET implant (81\% versus $67 \%$, statistically significant). Recognition of spondee words and sentences, however, was comparable, with no betweengroup differences.

Analysis of Carina implant recipients with the device switched on versus switched off showed a mean functional gain of $21.3 \mathrm{~dB}$ (range, 9.3 to $39.0 \mathrm{~dB}$ ). ${ }^{2}$ Interestingly, functional gain outcomes were superior in patients with conductive and mixed hearing loss versus sensorineural hearing loss $(28.2 \mathrm{~dB} \text { versus } 14.4 \mathrm{~dB})^{2}$

The possibility of use in any setting, cosmetic benefits, and absence of a microphone make the Esteem device a very 
appealing option. Kraus et $\mathrm{al}^{15}$ studied the results of a 12 month follow-up in 52 patients implanted with the Esteem device and found that the functional gain was $27 \pm 1 \mathrm{~dB}$ for 48 patients and 4 of them were stable at $\pm 10 \mathrm{~dB}$. Nevertheless, the implantation procedure is quite complex and long, and transducer size is a critical factor. ${ }^{2}$ The need for removal of the long process of the incus is another drawback, posing an additional risk of reduction in residual hearing if the device does not function properly. ${ }^{2}$

\section{Conclusion}

Implantable hearing aids, which are currently in the early stages of development, will unquestionably be the major drivers of advancement in otologic practice in the 21st century. With improvements in technology and a pressing need to improve the quality of life of an increasingly aged population, which will consequently require increasing levels of hearing support, these devices are bound to become more and more widespread.

\section{References}

1 Chen DA, Backous DD, Arriaga MA, et al. Phase 1 clinical trial results of the Envoy System: a totally implantable middle ear device for sensorineural hearing loss. Otolaryngol Head Neck Surg 2004;131(6):904-916

2 Klein K, Nardelli A, Stafinski T. A systematic review of the safety and effectiveness of fully implantable middle ear hearing devices: the carina and esteem systems. Otol Neurotol 2012;33(6):916-921

3 Sterkers O, Boucarra D, Labassi S, et al. A middle ear implant, the Symphonix Vibrant Soundbridge: retrospective study of the first 125 patients implanted in France. Otol Neurotol 2003;24(3):427-436

4 Cummings CW, Flint PW, Harker LA, et al. Surgically implantable hearing aids. In: Cummings Otolaryngology Head \& Neck Surgery. 4th ed. Philadelphia, PA: Mosby; 2005:3574-3600

5 Jenkins HA, Atkins JS, Horlbeck D, et al. Otologics fully implantable hearing system: phase I trial 1-year results. Otol Neurotol 2008; 29(4):534-541
6 Cremers CWRJ, O'Connor AF, Helms J, et al. International consensus on Vibrant Soundbridge ${ }^{\circledR}$ implantation in children and adolescents. Int J Pediatr Otorhinolaryngol 2010;74(11): 1267-1269

7 Colletti V, Carner M, Colletti L. TORP vs round window implant for hearing restoration of patients with extensive ossicular chain defect. Acta Otolaryngol 2009;129(4):449-452

8 Colletti L, Carner M, Mandalà M, Veronese S, Colletti V. The floating mass transducer for external auditory canal and middle ear malformations. Otol Neurotol 2011;32(1):108-115

9 Colletti V, Soli SD, Carner M, Colletti L. Treatment of mixed hearing losses via implantation of a vibratory transducer on the round window. Int J Audiol 2006;45(10):600-608

10 Hough JV, Matthews P, Wood MW, Dyer RK Jr. Middle ear electromagnetic semi-implantable hearing device: results of the phase II SOUNDTEC direct system clinical trial. Otol Neurotol 2002;23(6): 895-903

11 Hough JV, Dyer RK Jr, Matthews P, Wood MW. Semi-implantable electromagnetic middle ear hearing device for moderate to severe sensorineural hearing loss. Otolaryngol Clin North Am 2001; 34(2):401-416

12 Fully-Implantable Ossicular Stimulator MET. D109629. Rev. B Surgical Training Manual European Union. Boulder, Colorado: Otologics LLC; 2011

13 Yanagihara N, Suzuki J, Gyo K, Syono H, Ikeda H. Development of an implantable hearing aid using a piezoelectric vibrator of bimorph design: state of the art. Otolaryngol Head Neck Surg 1984;92(6): 706-712

14 Goode RL, Rosenbaum ML, Maniglia AJ. The history and development of the implantable hearing aid. Otolaryngol Clin North Am 1995;28(1):1-16

15 Kraus EM, Shohet JA, Catalano PJ. Envoy Esteem Totally Implantable Hearing System: phase 2 trial, 1-year hearing results. Otolaryngol Head Neck Surg 2011;145(1):100-109

16 Dyer RK Jr, Nakmali D, Dormer KJ. Magnetic resonance imaging compatibility and safety of the SOUNDTEC Direct System. Laryngoscope 2006;116(8):1321-1333

17 Martin C, Deveze A, Richard C, et al. European results with totally implantable carina placed on the round window: 2-year followup. Otol Neurotol 2009;30(8):1196-1203

18 Jenkins HA, Atkins JS, Horlbeck D, et al. U.S. Phase I preliminary results of use of the Otologics MET fully-implantable ossicular stimulator. Otolaryngol Head Neck Surg 2007;137(2):206-212 